\title{
Spontaneous neonatal pneumomediastinum
}

\author{
Kiran Kumar Balegar V. ${ }^{1}$, Nadia Badawi ${ }^{1}$, Arieta Fa'asalele ${ }^{2}$, Kathryn Carmo ${ }^{1,2}$ \\ ${ }^{1}$ Department of Neonatology, Royal Alexandra Hospital for Children, Westmead, Australia; \\ ${ }^{2}$ Newborn \& Paediatric Emergency Transport Service, Westmead, Australia. \\ Email: kiranbal@rediffmail.com;
}

Received 18 June 2011; revised 26 July 2011; accepted 26 August 2011.

\begin{abstract}
Radiolucency in the superior mediastinum may be caused by a congenital cystic mass or pneumomediastinum. Most cases of pneumomediastinum occur in preterm newborns with surfactant deficiency receiving treatment with positive pressure ventilation. Spontaneous pneumomediastinum in term neonates is also rarely reported. We report the outcome for a term neonate with respiratory distress who was transferred for surgical evaluation of a cystic superior mediastinal mass.
\end{abstract}

Keywords: Spontaneous Pneumomediastinum; Superior Mediastinal Mass; Neonate

\section{INTRODUCTION}

A male infant of 38 weeks gestation and birth weight of $3520 \mathrm{~g}$ was referred to The Children's Hospital at Westmead, Australia for the surgical management of a radiolucent superior mediastinal mass. The infant was born by elective caesarean section to a primiparous woman after an uncomplicated pregnancy, in a regional hospital. Apgar scores were 7 and 9 at one and five minutes, respectively. Shortly after birth he developed mild respiratory distress requiring treatment with head box oxygen. The respiratory distress worsened over the next 24 hours and oxygen requirement increased to 50\% although blood gas analysis and haemodynamics remained stable. Chest $\mathrm{x}$ ray at this stage revealed a radiolucent superior mediastinal shadow. He was commenced on CPAP and the state Newborn Emergency Transport Service (NETS) team was contacted regarding advice and transfer. Review of an $\mathrm{x}$-ray film through telemedicine and multidisciplinary discussion with the receiving neonatologist, transport team and cardiothoracic surgeon indicated the possibility of pneumomediastinum. It was agreed to discontinue the CPAP and transfer the baby by road in crib oxygen, rather than by air, despite the longer travel time. The follow-up chest $\mathrm{x}$-ray at the tertiary centre revealed a classical Spinnaker sail sign of a pneumomediastinum (Figure 1) with an otherwise normal appearance of the lung fields. Conservative management in head box oxygen resulted in complete resolution of the pneumomediastinum over the subsequent 72 hours (Figure 2).

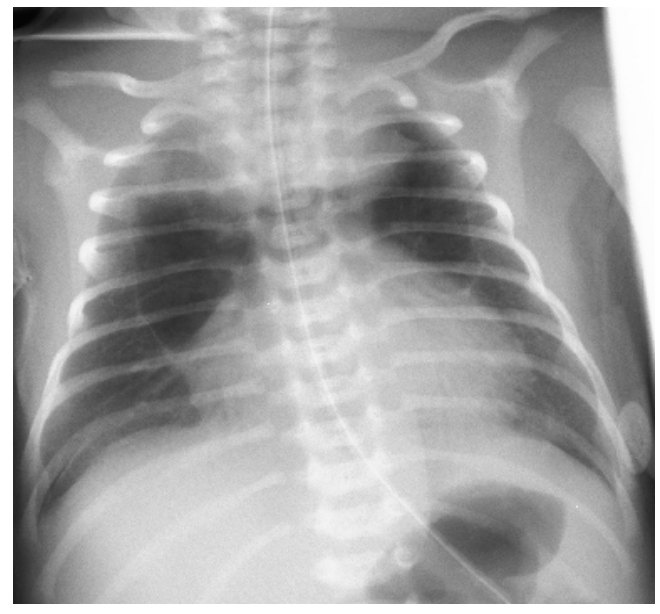

Figure 1. Chest x-ray on admission showing cystic appearance and Spinnaker sail sign of pneumomediastinum.

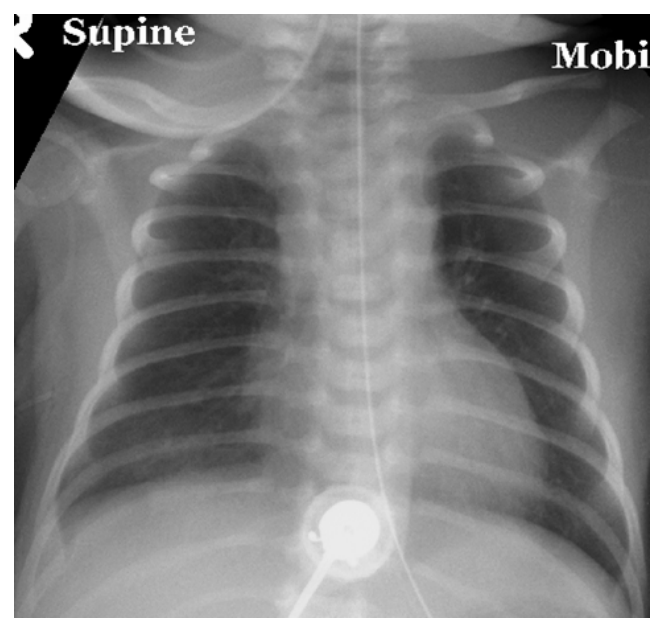

Figure 2. Chest $\mathrm{x}$-ray after 72 hours showing complete resolution of pneumomediastinum. 


\section{DISCUSSION}

Although most reported cases of neonatal pneumomediastinum ensue exposure to positive pressure in the setting of premature or diseased lungs [1-3], spontaneous pneumomediastinum that occurs in the absence of clearly defined precipitating factors, has rarely been reported $[4,5]$. Pathogenesis of pneumomediastinum was first demonstrated experimentally by Macklin in 1939 [6]. Exposure to an increased pressure gradient between the alveoli and the pulmonary interstitium results in alveolar rupture into the perivascular space, at the junctional area between compliant bronchial airway and poorly compliant alveoli. Air dissects along the perivascular sheaths towards the hilum and leaks into loose connective tissue spaces of the mediastinum. The usual triggering agent is positive pressure ventilation in combination with an underlying abnormality such as surfactant deficiency or meconium aspiration syndrome that renders the alveoli more fragile and prone to rupture [7]. In spontaneous pneumomediastinum the pressure gradient is thought to be generated by excessive negative intrapleural pressure during vigorous respiratory efforts in term infants, coupled with an uneven inflation of alveoli at birth [8]. This is the most plausible explanation in our infant.

Although chest roentgenography can be diagnostic in most cases of pneumomediastinum, the appearance may be confused with other surgical cystic mediastinal lesions such as congenital cystic adenomatoid malformation or congenital lobar emphysema, as illustrated in our case. This is because of the unique anatomical feature of the neonatal thymus characterized by a thick fascial capsule which envelopes the thymus and merges with the fibrous pericardium [8]. Air tends to loculate around the thymus and sometimes can even dissect within the interlobular and connective tissue septa of the thymic capsule, giving rise to a cystic appearance. It is postulated that this cystic appearance is uncommon in older children and adults because the thymus and its fascia undergo atrophy and are unable to contain a sudden air leak $[5,8]$.

Suspicion of pneumomediastinum prevented us from using aggravating factors such as CPAP or positive pressure ventilation. Between the 2 available options namely, transporting the baby in pressurised air transport vehicle at a lower altitude versus road transport - we chose the latter so as to avoid the possible disastrous effects from expansion of gases in the closed spaces at lower ambient air pressure (as dictated by Boyle's law).

A careful evaluation of the $x$-ray film in our case revealed the crescentic configuration of the elevated thymic lobes resembling a windblown Spinnaker sail. Serial $\mathrm{x}$-ray films showing evolution of the pneumome- diastinum with this classic Spinnaker sail sign followed by complete resolution avoided CT scan, which otherwise is the investigation of choice to confirm the nature of a mediastinal mass.

Most cases of spontaneous pneumomediastinum resolve spontaneously. Timely recognition and avoidance of aggravating factors constitute the crux of management and prevent deterioration and haemodynamic instability [7].

\section{KEY POINTS}

Neonatal pneumomediastinum can be confused with surgical cystic radiolucent mass owing to the unique anatomical feature of the neonatal thymus characterized by a thick enveloping fascial capsule that contains air.

Awareness of spontaneous pneumomediastinum in newborn babies with respiratory distress helps to direct the nature of respiratory support and the mode of transport. With timely recognition and avoidance of aggravating factors, most cases resolve spontaneously.

\section{REFERENCES}

[1] Lin, H.P., Johnson, R.O., Lam, K.L., Ong, T.H. and Singh, J. (1979) Pneumomediastinum in the newborn period and early childhood. Singapore Medical Journal, 20, 278-282.

[2] Rosenfeld, D.L., Cordell, C.E. and Jadeja, N. (1990) Retrocardiac pneumomediastinum: Radiographic finding and clinical implications. Pediatrics, 85, 92-97.

[3] Mohamed, I.S., Lee, Y.H., Yamout, S.Z., Fakir, S. and Reynolds, A.M. (2007) Ultrasound guided percutaneous relief of tension pneumomediastinum in a 1-day-old newborn. Archives of Disease in Childhood-Fetal and Neonatal Edition, 92, F458.

doi:10.1136/adc.2006.114322

[4] Lawal, T.A., Glüer, S. and Reismann, M. (2009) Spontaneous neonatal pneumomediastinum: The "spinnaker sail" sign. European Journal of Pediatric Surgery, 19, 50-52. doi:10.1055/s-2008-1038393

[5] Lee, C.T., Tsao, P.N. and Peng, S.S. (2008) Spontaneous multiseptated cystic pneumomediastinum in a term newborn. Pediatrics \& Neonatology, 49, 197-200. doi:10.1016/S1875-9572(09)60009-7

[6] Macklin, C. (1939) Transport of air along sheaths of pulmonic blood vessels from alveoli to mediastinal mediastinum. Archives of Internal Medicine, 64, 913-926.

[7] Hauri-Hohl, A., Baenziger, O. and Frey, B. (2008) Pneumomediastinum in the neonatal and paediatric intensive care unit. European Journal of Pediatrics, 167, 415-418. doi:10.1007/s00431-007-0517-9

[8] Low, A.S., Tan-Kendrick, A.P., Loh, M. and Chui, C.H. (2003) Spontaneous multiloculated multiseptated pneumomediastinum in a newborn baby: The spinnaker sail is rigged-CT features with pathologic correlation. Pediatric Radiology, 33, 712-715. doi:10.1007/s00247-003-0970-1 